\title{
COMPARATIVE ACTIVITIES OF NORADRENALINE AND ADRENALINE IN SOME BIOLOGICAL TESTS
}

\author{
THE RABBIT INTESTINE SEGMENT METHOD, \\ THE CAT PARADOXICAL PUPIL REACTION \\ AND THE DOG BLOOD PRESSURE
}

\author{
TATUZI SUZUKI, TERUO NAKAMURA AND CHIKAMASA NINAGAWA* \\ Department of Physiology, Nagasaki University School of Medicine, Nagasaki
}

Recently it is generally accepted that noradrenaline is contained along with adrenaline in the adrenal medulla as an active principle.

However, the suggestion of Kojima et al..(1) on the presence of some active principles allied with adrenaline in the adrenals appeared as long ago as 1932.

Taking the adrenaline hydrochloride of Sankyo Co. as the standard, they estimated the adrenaline equivalents in the extract of adrenal medulla or of whole adrenal gland of various species of animals. In their experiments some biological methods such as the cat paradoxical pupil reaction, the dog blood pressure, the rabbit intestine segment method, etc. and some chemical methods were used. The estimates of adrenaline varied according to the methods of estimation. With the extract of bovine and pig adrenal medulla or adrenal gland, the highest value of adrenaline equivalents was yielded by the rabbit intestine segment method and the smallest in the biological estimates by the cat paradoxical pupil reaction. The extract of horse adrenal gland gave similar values of adrenaline regardless of the method used. On the other hand, with the rabbit adrenal extract, the rabbit intestine segment method yielded the smallest value.

They assumed from their results that not only adrenaline but also some allied substances would be contained in the extract of medulla or whole gland of the adrenals and the amount of these active principles would be different according to the animal species. They suggested further that the effect of some active principles would be quantitatively different from that of adrenaline and in fact different according to the methods tested.

It is of interest to know whether the difference of adrenaline estimates of the adrenal extract is wholly due to the presence of two active principles, i.e. adrenaline and noradrenaline, and the difference of effects of two hormones in various test methods or not.

Along this line of question we have undertaken in the present investigation to compare quantitatively the activities of noradrenaline and adrenaline in some biological tests.

Received for publication March 8, 1953.

* 鈴木達二, 中村照夫, 䗄川親正 


\section{METHODS AND RESULTS}

In the present investigation the cat paradoxical pupil reaction, the dog blood pressure and the rabbit intestine segment method were applied. The synthetic $L$-noradrenaline- $D$-bitartrate-monohydrate (Sterling Winthrop), $D L$-noradrenaline (Sankyo) and $L$-adrenaline (Sankyo) were used. These were dissolved with $1 / 10 \mathrm{~N} \mathrm{HCl}$ as a $1: 1,000$ dilution and just before the experiments further diluted with $0.9 \%$ saline solution.

The amount of $L$-noradrenaline in $10.0 \mathrm{mg}$. of $L$-noradrenaline- $D$-bitartratemonohydrate was taken as $5.0 \mathrm{mg}$. In accordance with previous reports of some authors, the activity of $L$-noradrenaline was measured as twice that of the racemic one.

The cat paradoxical pupil reaction. Seven cats were used in this test. The procedure employed in our experiments was principally the same as that described by Sugawara (2) and that used in the experiments of Kojima et al. The superior cervical ganglion of the cat on one side was removed under ether anesthesia one week or more before the experiment. The test was performed under urethane anesthesia. The injection of adrenaline or noradrenaline solution was performed through a small cannula inserted in the femoral vein. The width of the pupil was measured in an accuracy to $1 / 8$ th of one millimeter, forming the image of the pupil by a lens on the scale.

In most of the cases the definite dilation of the pupil was caused by the injection of noradrenaline or adrenaline in a dose of $0.2-0.5 \mu \mathrm{g} . \quad L$-noradrenaline was in some instances less active than $L$-adrenaline, in others however their activities were about the same. The activity ratio of $L$-noradrenaline to $L$ adrenaline varied from $0.45: 1$ to $1.0: 1$, with a mean value of $0.73: 1$.

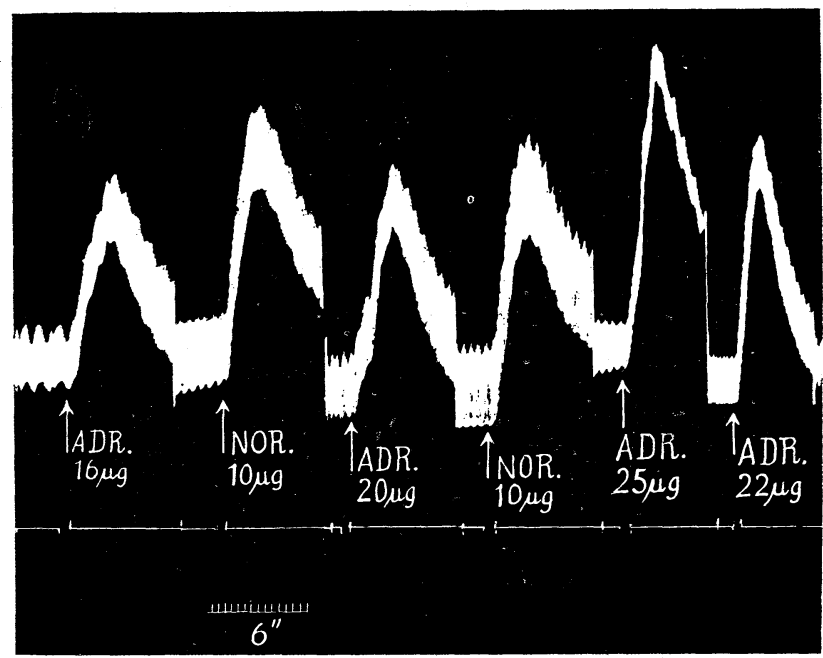

FIG. 1. Blood pressure of dog (Reduced to $3 / 5$ ). Non-anesthetized. Transected the spinal cord at the level of the lowest cervical segment. Both vagi cut. Intravenous injection of $L$-noradrenaline (NOR.) or $L$-adrenaline (ADR.). 
The blood pressure of dogs. Five dogs were used. Under ether anesthesia the spinal cord was transected at the level of $\mathrm{C}_{7}-\mathrm{C}_{8}$ and the ether was then removed immediately. About 2-3 hours thereafter the experiments were started without anesthesia. The blood pressure was registered by a Hg-manometer connected with the right femoral artery. In this test, $L$-noradrenaline was generally more active than $L$-adrenaline. $L$-noradrenaline in a dose of 3-15 $\mu$. or $L$-adrenaline in a dose of 5-25 $\mu \mathrm{g}$. was capable of elevating the blood pressure markedly. The activity ratio of noradrenaline to adrenaline was $1.6: 1-2.8: 1$ and the mean value was $2.1: 1$. As an example, the blood pressure tracing taken in one of these experiments is represented in fig. 1.

The rabbit intestine segment method. Intestinal segments of five rabbits were used.

The technique of this test was wholly the same as that described by Sugawara, Watanabe and Saito (3) and that used in the experiments of Kojima et al. The intestinal segment was cut off from the part near the opening of the pancreatic duct. The volume of the intestinal segment chamber was about $3 \mathrm{cc}$. In every case, atropine-Tyrode solution, in which the intestinal strip was moving rhythmically, was replaced by the diluted blood solution $(0.5 \mathrm{cc}$. of dog blood +2 cc. of Tyrode solution), which was then replaced by the diluted blood solution contained adrenaline or noradrenaline $(0.5 \mathrm{cc}$. of dog blood $+(2.0-2 a) \mathrm{cc}$. of Tyrode solution $+a \mathrm{cc}$. of double Tyrode solution $+a \mathrm{cc}$. of adrenaline or noradrenaline solution).

In our experiments $L$-noradrenaline was found to be strikingly more active than $L$-adrenaline. The activity ratio of noradrenaline to adrenaline was 2.0 : $1-3.5: 1$, and the average value was $2.8: 1$. An example of this test is depicted in fig. 2.

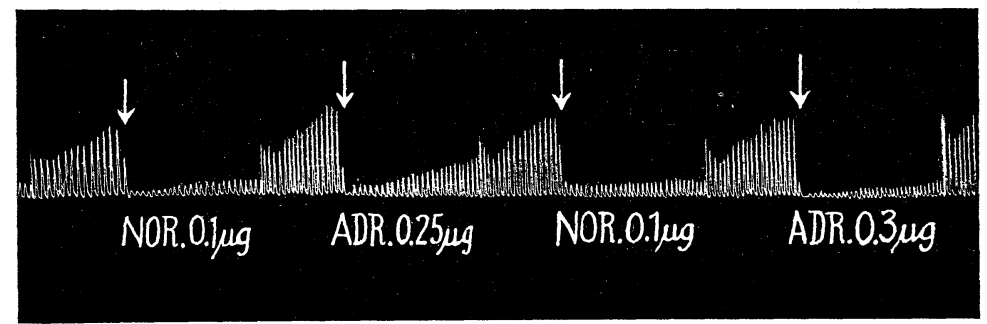

FIG. 2. Rabbit intestine tracing (Reduced to $3 / 4$ ). At the mark " $\downarrow$ " the diluted blood solution was displaced by the diluted blood solution containing $L$-noradrenaline (NOR.) or $L$-adrenaline (ADR.).

\section{DISCUSSION}

The biological effect of noradrenaline in comparison with that of adrenaline has been studied by many investigators. Here, only the reports of previous investigations, in which the same sorts of test-objects as ours were used, will be quoted.

As regards the effect of noradrenaline on the cat pupil, Greer et al. (4) 
observed that the pupil dilation caused by $D L$-noradrenaline was less than that by $L$-adrenaline and this difference of effect was not marked in the sensitized (sympathectomized) iris. In their studies, however, the comparison of activities was not performed quantitatively. In the paper of Euler (5) the activity ratio of noradrenaline to adrenaline was noted as $0.5-1: 1$, with which our results agree favorably.

The pressor effect of $D L$-noradrenaline in dogs under morphine-ether anesthesia was found by Schultz (6) to be 1.5 times as much as that of $D L$-adrenaline. In dogs under phenobarbital anesthesia, Tainter, Tullar and Luduena (7) found that the activity of noradrenaline is $164 \pm 10 \%$ of that of $L$-adrenaline. Luduena et al. (8) reported the activity ratio of noradrenaline to adrenaline in raising the blood pressure of barbitalized dogs as $1.7: 1$ in an average. In our experiments, the activity ratio of noradrenaline to adrenaline was found to be somewhat higher than the values reported by these previous investigators.

The effect of $L$-adrenaline on the isolated rabbit duodenum was found by Greer et al. to be several times more intensive than that of $D L$-noradrenaline. Gaddum, Peart and Vogt (9) obtained the value of 3 as the amount of $L$-noradrenaline which was equally active on the rabbit ileum with a unit amount of $L$-adrenaline. Holtz and Schümann (10) reported that adrenaline was twice as effective as noradrenaline on the rabbit ileum. The activity ratio of $D L$ noradrenaline to $L$-adrenaline on the rabbit ileum was measured by West (11) as $1: 2$. The same result was obtained by Takenaka, Namba and Hongo(12). Inasmuch as $D L$-noradrenaline is one half as effective as $L$-noradrenaline, the results of West and Takenaka $e$ t al. show that the effect of $L$-noradrenaline on the rabbit intestine is about the same as that of $L$-adrenaline. Using $L$-noradrenaline, Tainter, Tullar and Luduena found that the excised rabbit intestinal segment is relaxed by noradrenaline to about the same degree as by adrenaline. This was confirmed by Luduena et al. However, it was reported in a paper by Holton (13) that the effect of $D L$-noradrenaline on the rabbit duodenum is about the same as that of $L$-adrenaline. Her result indicates that $L$-noradrenaline is about twice as effective as $L$-adrenaline. Graham (14) noted that $L$-noradrenaline was more than twice as active as $L$-adrenaline on the isolated rabbit ileum. In our studies, $L$-noradrenaline was found to be strikingly more effective in comparison with $L$-adrenaline. The activity ratio was determined as $2.8: 1$ in a mean value and agreed roughly with the value of Graham.

After reviewing the previous information concerning the effect of noradrenaline on the isolated rabbit intestinal segment we noticed a disharmony of results. However, we can not decide here what is the responsible factor for such a difference.

Let us now discuss the discrepancy of adrenaline estimates of adrenal extract in various animal species reported by Kojima et al. In an extract of bovine adrenal medulla, the adrenaline equivalent was estimated by the rabbit intestine segment method, the dog blood pressure and the cat paradoxical pupil reaction as $15.5 \mathrm{mg} ., 12.4 \mathrm{mg}$. and $10.7 \mathrm{mg}$. per g. of tissue in a mean value, respectively. As the activity ratio of $L$-noradrenaline to $L$-adrenaline is the highest in the rabbit intestinal segment method, the smallest in the cat pupil method and the 
intermediate in the blood pressure method, such a discrepancy of adrenaline estimates as was observed by Kojima et al. may partly be explained by the presence of noradrenaline as well as adrenaline in the adrenal medulla.

However, to know whether the difference of adrenaline estimates is wholly due to the presence of noradrenaline and adrenaline or not, there is a need to compare the effect of adrenaline-noradrenaline mixture on some test objects with that of the adrenal extract.

The fact that a larger value is obtainable by the rabbit intestinal segment method than by the cat pupil method was also noted in the papers of Sugawara (15) (medullary tissue of cattle and pigs, whole adrenal gland of dogs and cats), of Aomura (16) (adrenals of dogs, goats and barger) and of Hatano (17) (bovine adrenal medulla). As a matter of fact Aomura suggested in his paper that various kinds of active principles besides adrenaline are involved in the adrenal extract and some of them act more strongly on the rabbit intestinal segment than others.

As regards horse adrenal extract, almost similar values were obtained by Kojima et al. with various tests, contrary to the adrenaline estimates in other species of animals. How to explain this similarity of values? It was suggested by Kojima et al. that horse adrenal extract may contain simply one kind of active principle. However according to the experimental result of Ozaki (18) in our Laboratory, noradrenaline was found to be contained besides adrenaline in horse medullary extract in an amount of $18 \%$ of the total active principles.

It may be suggested tentatively here that the adrenaline used in the experiments of Kojima et al. as the standard, contained noradrenaline in almost the same percentage as that of horse adrenal extract. In fact, Goldenberg et al. (19) found that some samples of epinephrine (U.S.P.) contained noradrenaline in $12-18.5 \%$. Similar findings were also obtained by Auerbach and Angell (20).

\section{SUMMARY}

The effects of $L$-noradrenaline and $L$-adrenaline on the denervated iris of cats, blood pressure of dogs and intestinal segments of rabbits were compared.

In the cat paradoxical pupil reaction, noradrenaline was equally effective or less effective in comparison with adrenaline. The activity ratio of noradrenaline to adrenaline varied from $0.45: 1$ to $1.0: 1$, with a mean value of $0.73: 1$.

Noradrenaline was more effective on dog blood pressure than adrenaline, and the ratio of activity of noradrenaline to that of adrenaline varied between $1.6: 1$ and $2.8: 1$, with a mean value of $2.1: 1$.

Noradrenaline was found to be more effective than adrenaline on rabbit intestinal segments. The activity ratio was $2.0: 1-3.5: 1$, with a mean value of $2.8: 1$.

The discrepancy of adrenaline estimates of adrenal extract yielded by various biological methods was discussed.

\section{REFERENCES}

1. Kojima, T., Nemoto, M., Saito, S., Sato, H. AND Suzuki, T. Tohoku J. Exp. Med. 19: 205, 1932. 
2. Sugawara, T. Tohoku J. Exp. Med. 8: 355, 1927.

3. SugaW ARA, T.. WATANabe, M. AND SaIto, S. Tohoku J. Exp. Med. $7: 6$ ff., 1926.

4. Greer, C. M., Pinkston, J. O., BaXter, JR. J. H. AND Brannon, E. S. J. Pharmacol. $60: 108,1937 ; 62: 189,1938$.

5. Euler, U. S. v. Erg. Physiol. $46: 281$ f., 1950.

6. Schultz, W. H. Hygienic Lab. Bull. No. 55, 31 ff., 1909.

7. Tainter, M. L., Tullar, B. F. ANd Luduena, F. P. Science 107: 39, 1948.

8. Luduena, F. P., Ananenko, E., Siegmund, O. H. AND Miller, L. C. J. Pharmacol. $95: 155,1949$.

9. Gaddum, J. H., Peart, W. S. And Vogt, M. J. Physiol. 108: 467, 1949.

10. HOLTZ, P. AND SchÜMANN, H. J. Nature 165: 683, 1950.

11. West, G. B. J. Physiol. 106: 418, 1947.

12. TAKenAKA, F., NAMBA, K. AND HoNGO, T. Nagasaki Igk. Z. 27 : 618, 1952 (Japanese).

13. Holton, P. J. Physiol. 108: 525, 1949.

14. GRAHAM, J. D. P. J. Physiol. 108: 15 P, 1949.

15. SUgAWARA, T. Tohoku J. Exp. Med. 11: 410, 1928.

16. Aomura, T. Tohoku J. Exp. Med. 14: 291, 1929.

17. Hatano, M. Tohoku J. Exp. Med. 29: 307, 1936.

18. OzAKI, T. will be published in Tohoku J. Exp. Med.

19. Goldenberg, M., Faber, M., Alston, E. J. AND Chakgaff, E. C. Science 109 : 534, 1949.

20. AUerbach, M. E. AND ANGell, E. Science 109: 537, 1949. 